\title{
Rapid Microwave-Assisted Synthesis of Modified Pyrimidine and Purine Pyranonucleosides as Novel Cytotoxic, Antiviral Agents and Glycogen Phosphorylase B Inhibitors
}

\author{
Manta $\mathbf{S}^{1}$, Dimopoulou $\mathbf{A}^{2}$, Kollatos $\mathbf{N}^{2}$, Kyrkou $\mathbf{G}^{2}$, Spanos $\mathbf{S}^{2}$ and Komiotis $\mathbf{D}^{2 *}$ \\ ${ }^{1}$ Department of Chemistry, University of Cyprus, Nicosia, Cyprus \\ ${ }^{2}$ Department of Biochemistry and Biotechnology, University of Thessaly, Larissa, Greece
}

\begin{abstract}
Nucleosides take an important place in medicinal chemistry as the structural basis for the development of therapeutic agents. The chemistry of substituted base-modified nucleosides has been an interesting field of study over the last two decades owing to their biological properties. This mini-review summarizes recent efforts on the synthesis of C5- and C8-alkynyl base-modified pyranonucleoside analogues using Sonogashira cross-coupling reaction under microwave irradiation.
\end{abstract}

Keywords: Sonogashira reaction; Base-modified; Pyranonucleosides; Microwave irradiation; Cytotoxic/antiviral activity

\section{Introduction}

Several purine-and pyrimidine-substituted nucleosides exhibit activity in both solid tumors and hematological malignancies, behaving as antimetabolites, competing with physiological nucleosides, and consequently, interacting with a large number of intracellular targets to induce cytotoxicity [1]. Among them, alkynyl-modified uridines exhibited significant antiviral [2-4] and anticancer activities [4]. e.g., the internal aromatic-substituted alkyne $p$-tolylethynyl-2'-deoxyuridine showed high potency against MCF-7 (IC $0.9 \pm 0.2 \mu \mathrm{M}$ ), comparable to 5-fluorouracil and Cisplatin [5] while 5- ethynyl-2'-deoxyuridine, was the most potent inhibitor against MCF-7 and MDA-MB-231 human breast cancer cells ( $\mathrm{IC}_{50} 0.4 \pm 0.3$ and $4.4 \pm 0.4 \mu \mathrm{M}$, respectively), the same compound also inhibited the replication of HSV-1, HSV-2 (herpes simplex virus type 1,2 ) and VV (vaccinia virus) at concentrations of 0.1-1 $\mu \mathrm{g} / \mathrm{mL}[6,7]$. Little effort has been made towards the synthesis of C8-modified purine nucleosides, nevertheless, some interesting biological properties have been reported: e.g., selected 8-alkynyl adenosines were selective ligands for the $\mathrm{A}_{3}$ adenosine receptor subtype behaving as adenosine antagonists [8], and various $C 8$-modified 2'-deoxy adenosines induced delayed chain termination in vitro and showed moderate anti HIV-1 activity in cell culture [9]. Considering the progress made in this direction, the present mini review presents an update of recent developments on pyrimidine- and purine-modified pyranonucleosides that possess interesting biological properties. In particular, the molecular design, synthesis and biological activity of C5-alkynyl pyrimidines and C8-alkynyl purine pyranonucleosides is presented. Aiming at a more detailed structure-activity relationship studies, a variety of alkyne substituents $\mathrm{R}$ are reported, such as linear alkyl chains and arenes substituted with linear and branched alkyl groups.

\section{Results and Discussion}

In 2013, numerous novel C5-alkynyl uracil and cytosine glucopyranonucleosides were first synthesized and biologically evaluated [10]. Analogues 3a,b-9a,b, 11a and 12a were prepared via their iodinated precursors $\mathbf{2 a}$ and $\mathbf{2} \mathbf{b}$ using $\operatorname{Pd}(0)$-catalyzed Sonogashira cross-coupling reactions under microwave irradiation (Figure 1). When compared to conventional heating [11], the MW technology completed the synthesis much faster, while the yields of the products were slightly increased (by 3-13\%). All the newly synthesized compounds were evaluated for their cytostatic activity against human cervix carcinoma (HeLa), human lymphocytes (CEM) as well as murine leukemia (L1210) cells. The 5-substituted uracil pyranonucleosides showed superior antiproliferative activity to their cytosine counterparts. Among the nucleosides tested, the phenylethynyl uracil pyranonucleoside derivative 5a, effectively inhibited tumor cell proliferation ( $\mathrm{IC}_{50} 5.2-6.2 \mu \mathrm{M}$ ) similar to that of 5-fluorouracil ( $\mathrm{IC}_{50}$ 0.33-18 $\mu \mathrm{M})$, whereas its cytosine congener showed no appreciable cytostatic action $\left(\mathrm{IC}_{50} 201 \rightarrow 250 \mu \mathrm{M}\right)$. The cyclization of $\mathbf{3 a}$ and $\mathbf{4 a}$ to the target bicyclic nucleosides 10a and 11a achieved through extended reaction time (irradiation for $8 \mathrm{~min}$ ) and it was based on a 5-endo-dig electrophilic cyclization $v i a$ an $O$-hetero-annulation process. Kinetic studies also showed that 1-( $\beta$ - $D$-glucopyranosyl)-5-ethynyluracil 9a was the best glycogen phosphorylase b (GPb) inhibitor (Ki $4.7 \mu \mathrm{M})$. Crystallography revealed that inhibitors with a long C5-alkynyl group exploited interactions with the b-pocket of the active site and induced significant conformational changes of the 280 s loop compared to $\mathrm{GPb}$ complex with compounds hosting a short $C 5$-alkynyl group. The results highlight the importance in the length of the aliphatic groups used to enhance inhibitory potency for the exploitation of the hydrophobic b-pocket. The most active inhibitor also had a moderate effect on glycogenolysis at the cellular lever with an $\mathrm{IC}_{50}$ value of $291.4 \mu \mathrm{M}$.

Recently, C5-aryl ethynyl uracil glucopyranonucleosides 13,15 and C5-arylethyl uracil pyranonucleosides $\mathbf{1 7 a}, \mathbf{e}, \mathbf{i}, \mathbf{j}, \mathbf{k}$ were also prepared [12] and evaluated for their cytostatic and antiviral activities. The protected analogues 12a,e,i,j (Figure 2) showed better cytostatic activity against human lymphocyte CEM tumor cells ( IC $_{50} 18-42$ $\mu \mathrm{M})$ compared to their unprotected counterparts $\mathbf{1 3} \mathbf{a}, \mathbf{e}, \mathbf{i}, \mathbf{j}$ which did not enhance growth inhibition of CEM cells $\left(\mathrm{IC}_{50}>250 \mu \mathrm{M}\right)$, while derivatives $\mathbf{1 7 a}, \mathbf{e}, \mathbf{i}, \mathbf{j}, \mathbf{k}$ were devoid of significant cytostatic activity $\left(\mathrm{IC}_{50}\right.$ 94-250 $\mu \mathrm{M}$ ). Their antiviral activities were measured against a large

*Corresponding author: Komiotis D, Laboratory of Bioorganic Chemistry, Department of Biochemistry and Biotechnology, University of Thessaly, Viopolis, 41500 Larissa, Greece, Tel: 302410565290; E-mail: dkom@bio.uth.gr

Received April 27, 2017; Accepted May 05, 2017; Published May 10, 2017

Citation: Manta S, Dimopoulou A, Kollatos N, Kyrkou G, Spanos S, et al. (2017) Rapid Microwave-Assisted Synthesis of Modified Pyrimidine and Purine Pyranonucleosides as Novel Cytotoxic, Antiviral Agents and Glycogen Phosphorylase B Inhibitors. Med Chem (Los Angeles) 7: 865-868. doi: 10.4172/2161-0444.1000443

Copyright: ( 2017 Manta S, et al. This is an open-access article distributed under the terms of the Creative Commons Attribution License, which permits unrestricted use, distribution, and reproduction in any medium, provided the original author and source are credited. 
Citation: Manta S, Dimopoulou A, Kollatos N, Kyrkou G, Spanos S, et al. (2017) Rapid Microwave-Assisted Synthesis of Modified Pyrimidine and Purine Pyranonucleosides as Novel Cytotoxic, Antiviral Agents and Glycogen Phosphorylase B Inhibitors. Med Chem (Los Angeles) 7: 865868. doi: 10.4172/2161-0444.1000443
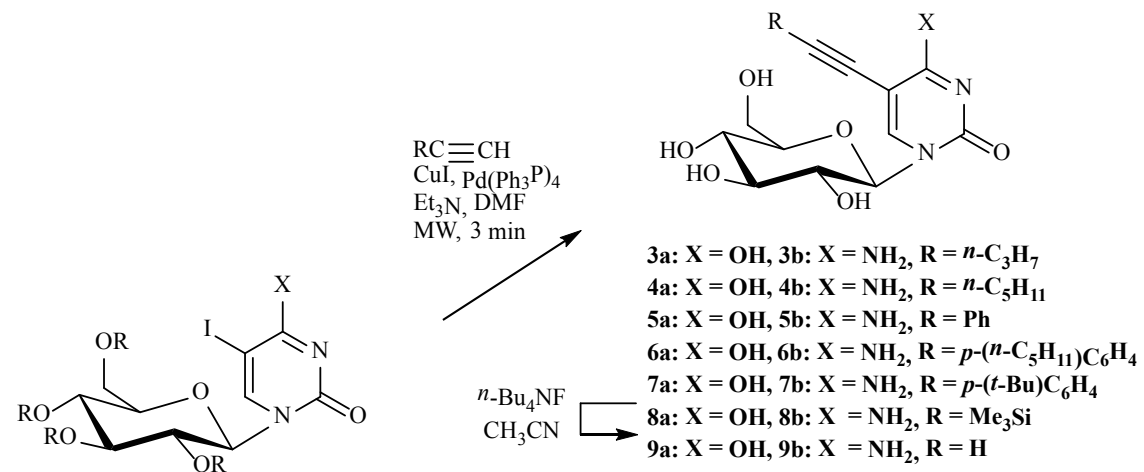

$\mathrm{NH}_{3}$

1a: $R=A c, X=O H 1 b: R=A c, X=\mathrm{NH}_{2}$

$\mathrm{MeOH}$

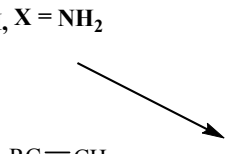

$\mathrm{RC} \equiv \mathrm{CH}$

$\mathrm{CuI}, \mathrm{Pd}\left(\mathrm{Ph}_{3} \mathrm{P}\right)_{4}$

$\mathrm{Et}_{3} \mathrm{~N}, \mathrm{DMF}$

MW, 8 min

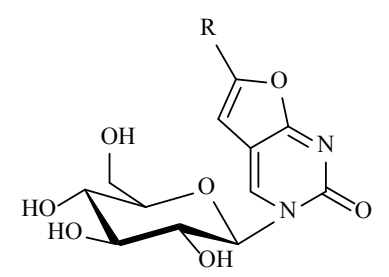

10a: $\mathbf{R}=n-\mathrm{C}_{3} \mathrm{H}_{7}$

11a: $\mathrm{R}=n-\mathrm{C}_{5} \mathrm{H}_{11}$

Figure 1: Synthesis of C5-alkynyl pyrimidine glucopyranonucleosides.

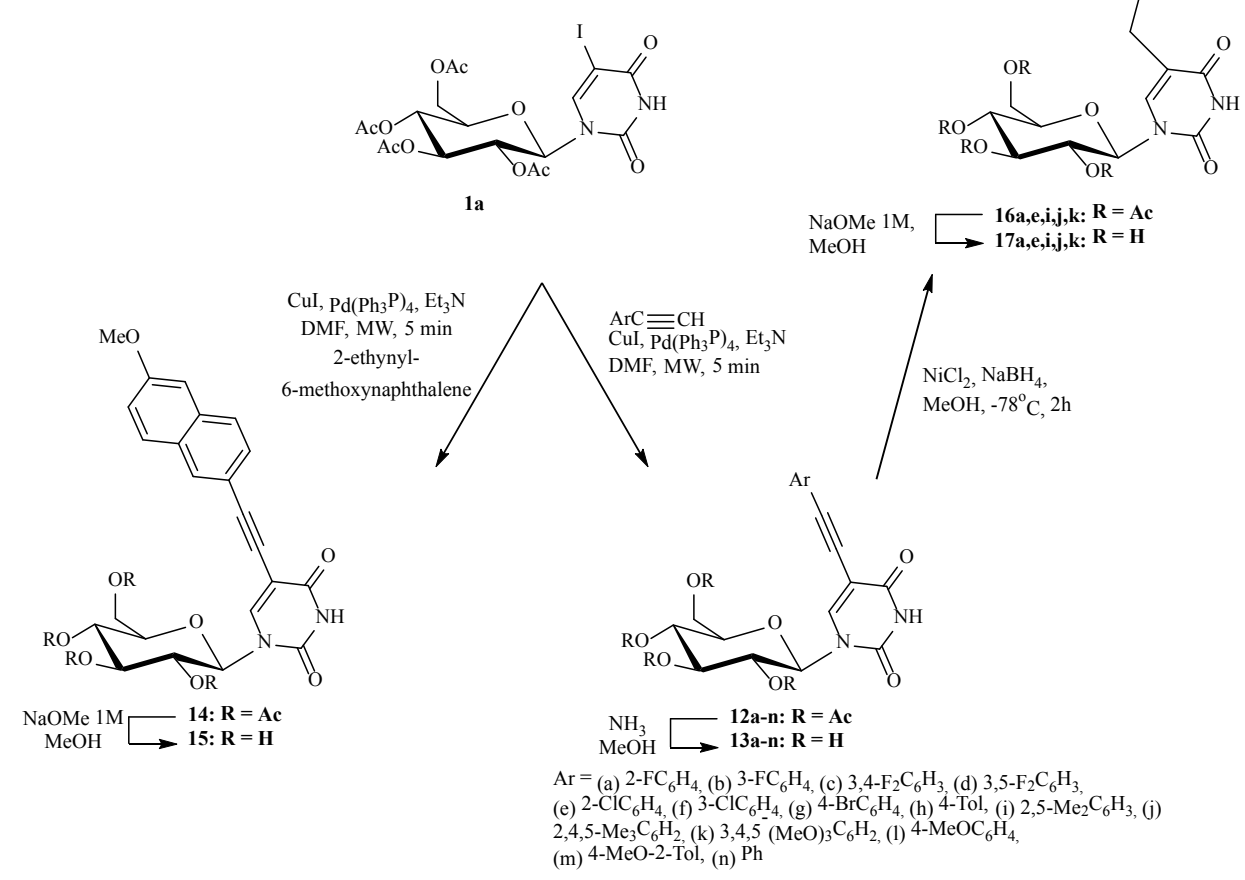

Figure 2: Synthesis of $C 5$-arylalkynyl uracil glucopyranonucleosides and of 1-( $\beta$-D-glucopyranosyl)- 5-[arylethyl]uracil.

number of DNA and RNA viruses including herpes simplex virus type 1,2 (HSV-1,2), vaccinia virus (VV), adeno-virus 2 (Ad-2) and human coronavirus 229E (HCoV-229E). Only analogue 17i exhibited the best anti-VV activity $\left(\mathrm{EC}_{50} 10 \mu \mathrm{M}\right)$ : 10 - and 25-more active than the reference drugs Ganciclovir (CMV) $\left(\mathrm{EC}_{50} 100 \mu \mathrm{M}\right)$ and Acyclovir (ACV) $\left(\mathrm{EC}_{50} 250 \mu \mathrm{M}\right)$, respectively, on HEL cell culture. Novel C5arylalkynyl uracil pyranonucleosides bearing 3 '-C-trifluoromethyl$\mathrm{D}$-allose as sugar moiety were recently synthesized via microwave- 
Citation: Manta S, Dimopoulou A, Kollatos N, Kyrkou G, Spanos S, et al. (2017) Rapid Microwave-Assisted Synthesis of Modified Pyrimidine and Purine Pyranonucleosides as Novel Cytotoxic, Antiviral Agents and Glycogen Phosphorylase B Inhibitors. Med Chem (Los Angeles) 7: 865868. doi: $10.4172 / 2161-0444.1000443$

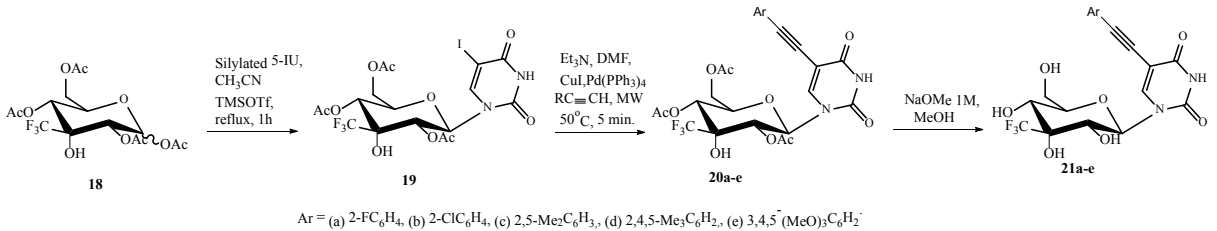

Figure 3: Synthesis of 1-(3'-C-trifluoromethyl- $\beta$ - $D$-glucopyranosyl)-5-[arylethynyl]uracil.
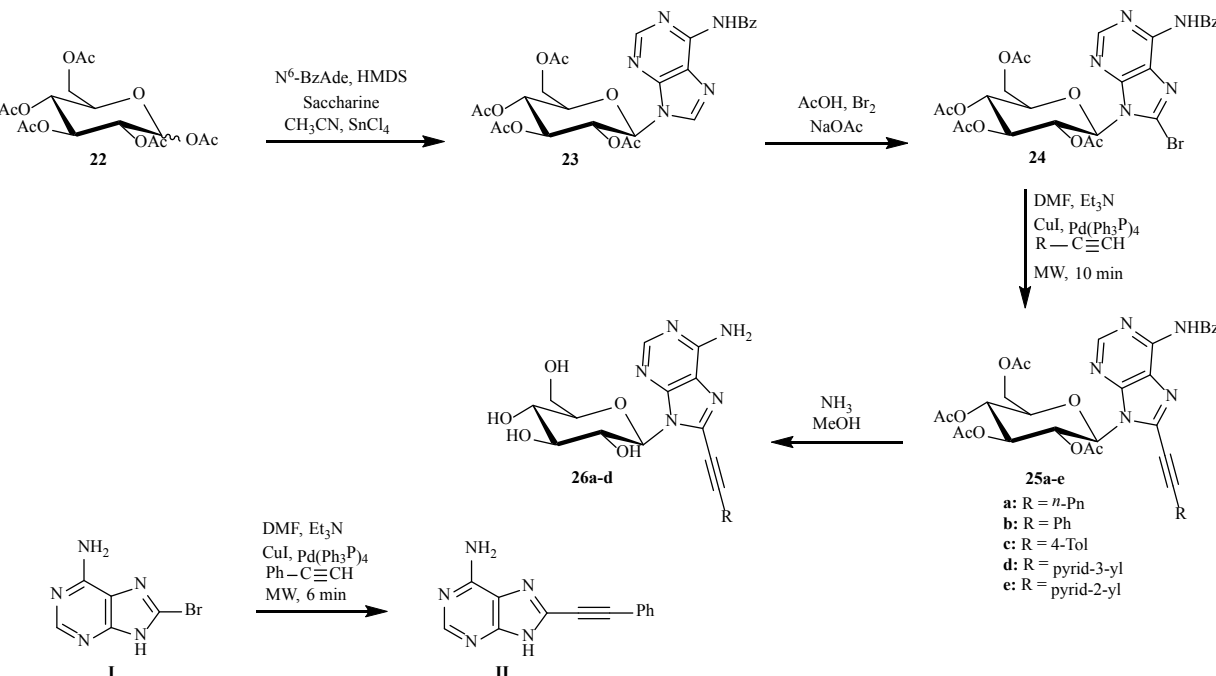

Figure 4: Synthesis of C8-alkynyl adenine glucopyranonucleosides and 8-phenylethynyl-adenine.

assisted Sonogashira coupling and were biologically evaluated [12]. The protected nucleosides $\mathbf{2 0 c}, \mathbf{d}$ proved to be cytotoxic against human lymphocyte $(\mathrm{CEM})$ cells $\left(\mathrm{IC}_{50} 19-20 \mu \mathrm{M}\right)$ similarly to 5 -fluorouracil $\left(\mathrm{IC}_{50} 0.33-18 \mu \mathrm{M}\right)$, contrary to their unprotected analogues $21 \mathbf{c}, \mathbf{d}$ which proved to be inactive $\left(\mathrm{IC}_{50}>178 \mu \mathrm{M}\right.$ ) (Figure 3).

Finally, the synthesis of the first purine glucopyranonucleosides $C 8$ alkynyl adenines 25, 26 and 8-phenyl-ethynyl-adenine (II) itself were also reported (Figure 4) [13] using the Sonogashira cross-coupling reaction. The cytostatic potential of compounds $25 \mathbf{e}\left(\mathrm{IC}_{50} 2.9-5.9\right.$ $\mu \mathrm{M})$ and $\mathrm{II}\left(\mathrm{IC}_{50} 3.0-10.0 \mu \mathrm{M}\right)$ was an order of magnitude lower than 5-fluorouracil ( $\mathrm{IC}_{50} 0.33-0.54 \mu \mathrm{M}$ ) on murine leukemia (L1210), and human cervix carcinoma (HeLa) cells, while the same compounds $\left(\mathrm{IC}_{50} 1.2-4.2 \mu \mathrm{M}\right)$ were more active than 5-fluorouracil $\left(\mathrm{IC}_{50} 18 \mu \mathrm{M}\right)$ on human lymphocyte (CEM) cells.

\section{Conclusion}

In the present review, we focused our attention on the relatively new literature data, concerning the $C 5$ - and $C 8$-alkynyl base-modified glucopyranonucleosides, as well as 8-phenylethynyl-adenine itself, via Sonogashira coupling conditions under microwave irradiation. With chemists becoming increasingly interested in biology, the demand for novel bioactive compounds with improved therapeutic potential is becoming high. Moreover, extensive structure-activity relationship studies of the novel pyranonucleoside analogues concerning different substituents in the aromatic ring as well as more detailed investigations on the molecular drug targets are envisaged.

\section{Acknowledgements}

This work was supported in part by the Postgraduate Program "Toxicology", Department of Biochemistry and Biotechnology, University of Thessaly.

\section{References}

1. Hatse S, De Clercq E, Balzarini J (1999) Role of antimetabolites of purine and pyrimidine nucleotide metabolism in tumor cell differentiation. Biochemical Pharmacology 58: 539-555.

2. De Clercq E, Descamps J, Balzarini J, Giziewicz J, Barr PJ, et al. (1983) Nucleic acid related compounds. 40. Synthesis and biological activities of 5-alkynyluracil nucleosides. J of medicinal chemistry 26: 661-666.

3. Efange SM, Cheng YC, Bardos TJ (1985) Synthesis and biological activities of 2-Pyrimidinone Nucleosides. III. 1, 2 5-Alkynyl-2-Pyrimidinone 2'-Deoxyribosides. Nucleosides \& Nucleotides 4: 545-564.

4. Vincent $P$, Beaucourt JP, Pichat L, Balzarini J, De Clercq E (1985) Syntheses, Activites Biologiques et Etude Conformationnelle D'Alcynyl-5 Desoxy-2 Uridines. Nucleosides \& Nucleotides 4: 429-445.

5. Meneni S, Ott I, Sergeant CD, Sniady A, Gust R, et al. (2007) 5-Alkynyl-2'deoxyuridines: chromatography-free synthesis and cytotoxicity evaluation against human breast cancer cells. Bioorganic \& medicinal chemistry 15: 3082 3088 .

6. De Clercq E, Descamps J, De Somer P, Barr PJ, Jones AS, et al. (1979) (E)5-(2-bromovinyl)-2'-deoxyuridine: A potent and selective anti-herpes agent Proceedings of the National Academy of Sciences 76: 2947-2951.

7. De Clercq E, Descamps J, Verhelst G, Walker RT, Jones AS, et al. (1980) Comparative efficacy of antiherpes drugs against different strains of herpes simplex virus. Journal of Infectious Diseases 141: 563-574.

8. Volpini R, Costanzi S, Lambertucci C, Vittori S, Klotz KN, et al. (2001) Introduction of alkynyl chains on C-8 of adenosine led to very selective antagonists of the A 3 adenosine receptor. Bioorganic \& medicinal chemistry letters 11: 1931-1934. 
Citation: Manta S, Dimopoulou A, Kollatos N, Kyrkou G, Spanos S, et al. (2017) Rapid Microwave-Assisted Synthesis of Modified Pyrimidine and Purine Pyranonucleosides as Novel Cytotoxic, Antiviral Agents and Glycogen Phosphorylase B Inhibitors. Med Chem (Los Angeles) 7: 865868. doi: $10.4172 / 2161-0444.1000443$

9. Vivet-Boudou V, Isel C, Sleiman M, Smyth R, Gaied NB, et al. (2011) 8-Modified-2'-deoxyadenosine analogues induce delayed polymerization arrest during HIV-1 reverse transcription. PloS ONE 6: e27456.

10. Dimopoulou A, Manta S, Kiritsis C, Gkaragkouni DN, Papasotiriou I, et al. (2013) Rapid microwave-enhanced synthesis of C5-alkynyl pyranonucleosides as novel cytotoxic antitumor agents. Bioorganic \& medicinal chemistry letters 23: $1330-1333$

11. Kantsadi AL, Manta S, Psarra AM, Dimopoulou A, Kiritsis C, et al. (2012) The binding of C5-alkynyl and alkylfurano [2, 3-d] pyrimidine glucopyranonucleosides to glycogen phosphorylase b: synthesis, biochemical and biological assessment. European Journal of Medicinal Chemistry 54: 740-749.

12. Dimopoulou A, Kollatos N, Manta S, Panagiotopoulou A, Karastergiou A, et al (2017) Facile microwave-assisted synthesis of various C5-modified pyrimidine pyranonucleosides as potential cytotoxic antitumor agents. Curr Microwave Chem.

13. Dimopoulou A, Manta S, Parmenopoulou V, Kollatos N, Christidou O, et al. (2015) An easy microwave-assisted synthesis of C8-alkynyl adenine pyranonucleosides as novel cytotoxic antitumor agents. Frontiers in Chemistry. 